\title{
Interconnectivity of Big Data Sources: Exploring Synergistic Relationships among Data
}

\author{
Johannes Weibl \\ LMU Munich \\ weibl@bwl.lmu.de
}

\begin{abstract}
In recent years, organizations manage an increasing amount of data in order to make better decisions, personalize products, or sell data. By data being combined from various sources, data assets interact with each other. When the interactions are synergistic, they create greater benefits than the sum of the value of the individual data assets. This study explores enablers, mechanisms, and potential outcomes of synergistic interactions among data assets. Based on systems theory and a synthesis of relevant synergy literature, I developed an initial synergy framework in a data context. On this basis, I conducted 14 qualitative interviews to assess the validity of my initial framework. The interview results assisted me in refining and contextualizing a unified conceptual framework of data synergies. The paper reveals that compatibility and contextual relatedness as enablers and informational complementary as a mechanism can lead to super-additive information value among data assets.
\end{abstract}

\section{Introduction}

Advances in IT technologies in recent years have increased the volume, variety, and velocity of data being generated, captured, and stored $[1,2]$ and the resulting data is often termed as "big data" [3]. Hereby data variety is often seen as the major driver for generating additional value [4]. The combining and interaction of heterogeneous data from various data sources especially facilitates companies in generating additional business value, for example, innovating their products and processes [5]. In the case of Netflix, for instance, it enables a whole new value proposition by combining user data to inform the content of their new series [6].

Information Systems (IS) research has begun to explain how business value is generated for organizations from big data in general $[7,8]$ and by referring to data variety in particular [9]. The possibility to synthesize data from various big data sources is often termed as "interconnectivity", which allows actors deeper insights from their combination $[9,10]$. Other researchers state that synthesized data have a greater value than the sum of its individual parts that enables analysts and decision makers to arrive more insights by exploring potential connections and leading to a better decision-making process in the end [11].

However, it still remains unclear how the underlying effects by which data assets interact with each other to generate additional value can be conceptualized. When data from various data sources are combined and synthesized, data assets synergistically interact with each other resulting in, for example, better decisions, personalized products, or the sale of the enriched data sets [1, 5]. Therefore, synergistic interaction among data can be an important source of organizational benefit, summarized as data synergies. None of the previously cited studies considers the synergistic interaction of data as the main source for generating new insights and additional value in organizations. Accordingly, the underlying effects and mechanisms through which heterogeneous data sources interact and synergize with each other are not well understood. Moreover, the concept of synergy is rarely used in IS literature [12], and its underlying mechanisms and potential benefits remain largely unexplored. Thus, this paper addresses the following research question:

How can the synergistic interaction among data assets be conceptualized?

To answer the research question, I developed a conceptual data synergy model that sheds light on the enabling conditions and underlying mechanisms of interconnected data assets. This model draws on the generic synergy concept in IS literature [12-14] and fosters a better understanding of what synergy is in a data context and how data assets, a relevant subset of IT resources [15], can create additional value. In a second step, I conducted 14 semi-structured expert interviews and applied a thematic analysis. As a result, 
I was able to validate, refine, and contextualize my initial data synergy concept.

By adapting the concept of synergy to the data context, this paper makes important contributions to both theory and practice. The study sheds light on the synergistic value generation from data variety. The paper reveals that data synergy consists of three factors: Enablers, Mechanism and Realization. Hereby compatibility and contextual relatedness are the two crucial enablers to leverage synergistic interactions among data sources and informational complementarity as the underlying mechanism can lead to super-additive information value. For practitioners the synergy framework provides a clear guideline to leverage benefits from various data sources in the era of big data. To utilize additional value from various big data sources, these data sources have to be compatible with existing data systems and their data have to have a contextual relationship with the stored information of their existing systems.

The paper is structured as follows. In section 2, I introduce the study's theoretical background (systems theory and concept of synergy). I then describe the two-step research methodology (section 3). Then I present my results based on literature (chapter 4) as well as validated and refined with interviews (chapter 5). In the discussion section (chapter 6), I discuss my revised and final conceptual framework of data synergies. Finally, I suggest implications for research and practice and lay out the limitations of my research.

\section{Theoretical background}

\subsection{Systems theory and synergy}

The term "synergy" is derived from the Greek word "synergos," meaning "working together." Synergy is the combined effort of resources to generate outcomes that are greater than the additive impact of each individual resource [12]. The interaction of individuals in a team, for instance, can lead to the establishment of synergistic knowledge within the group [16]. Typically, synergy is associated with positive outcomes [13, 14]. Nevertheless, it can have a negative effect when one resource degrades the effect of another resource, leading to fewer outcomes in total. This is often described as a substitution relationship [17].

The concept of synergy is based on the following assumption: "Synergy is meaningful only if there are at least two interacting resources" [12]. Furthermore, the concept of synergy addresses typical limitations of the resource-based view (RBV) of an organization [18] in explaining the business value of IT resources. RBV theory typically conceptualizes organizational resources in isolation and therefore underrates their business value. Systems theory, in contrast, conceptualizes the interaction among organizational resources, which creates greater value [13, 14].

Systems theory is the theoretical underpinning behind the concept of synergistic resources [12] and conceptualizes systems as a whole, instead of merely as individual parts [19]. A system is "a composite thing comprising a number of subsystems, which interact to accomplish a set of goals" [12]. A whole system, derived from the synergistic interaction of its parts, results in the sum of the parts plus their interactions [19]. The synergistic interactions lead to positive emergent properties, which may add to a system's goal [20]. An organization in regard to systems theory, for example, can be seen as a set of interconnected respective subsystems [21]. This perspective is in contrast to the RBV, which views an organization exclusively as a bundle of resources [18]. The application of systems theory helps to conceptualize the interaction and synergy among resources, which extends the explanatory power of the RBV theory. Analogous to Someh and Shanks [12], I use the terms "systems" and "resources" interchangeably in this study.

Synergy has been conceptualized and used in a diverse range of IS research streams and at multiple levels of analysis. The range of IS literature concerning synergy reaches from the business value of IT assets $[13,22]$, to IT value co-creation [23], to knowledge management [24] and human resource management [25], among others. Regarding the level of analysis, synergy has been explored, for example, at the interorganizational level [26]. Synergy enhances the value co-creation of multiple organizations. Further, researchers have investigated the synergy effects between various functional or business areas within an organization [14], on an individual level between individuals [16], and between individuals and their technologies [12, 13]. However, in spite of the many theoretical explanations about synergy, most of these studies do not focus on synergy. Instead, they assess the synergistic effects between variables rather than deriving and contextualizing a distinct synergy construct. Nevo and Wade [13] and Someh and Shanks [12] constitute the exception in that they develop a conceptual framework of synergy in IS.

\subsection{The concept of synergy in IS literature}

Several researchers have discussed the concept of synergy in IS literature, amongst the most cited is that of Nevo and Wade [13], who present a holistic view of synergy comprising several factors that enable certain conditions and mechanisms leading to the realization 
of synergistic outcomes (see Figure 1). Nevo and Wade's work and its extension by Someh and Shanks [12] help to structure and analyze my insights regarding the synergy effects among data assets:

Synergy Enablers: Nevo and Wade [13] state that synergy might be difficult to achieve without the presence of enabling conditions. Enablers support and facilitate the progress of synergistic interaction among resources and influence the benefits of their relationship. As mentioned in various literature streams, the most crucial enabler is compatibility between resources [13, 25, 27]. According to systems theory, the synergistic relationship depends upon the mutual compatibility of its components [28]. Components are compatible "when certain properties match or are in alignment" [13]. Thus, compatibility refers to "the degree that systems fit with each other and are in alignment [...] and are able to seamlessly work with each other" [12]. Conversely, IT assets may be interpreted as incompatible when they must be intensely modified before interactions among them can be observed [13].

Synergy Mechanisms: The synergy mechanisms occur among resources (e.g., IT assets) to realize their potential. It refers to activities through which resources are combined to enlarge and complement each other's functionalities and efficiency. This activity is often described as the complementary mechanism, which has its roots in strategy and economics literature and is theoretically grounded in the economic theory of complementarities [29]. According to the economic theory of complementarities, "a set of resources is complementary when the returns from a resource vary in relation to the levels of return from other resources" [12].

Synergy Realization: Synergy is realized when outcomes are greater than the sum of the value of their individual parts. Through the theoretical lens of systems theory, the interaction among resources enables the emergence of new properties that cannot be led back to individual resources [13]. Positive emergent properties facilitate additional leverage superior to the independent effects of each resource in isolation [12]. This implies that an increase of the value of any of the resources enhances the value and return of the other resources [14]: Value $(A+B)>$ Value (A) + Value (B). Tanriverdi [14] further concludes that the complementarity among resources can be used to create super-additive value synergies, referring to the phenomenon that the joint effect of resources is greater than the sum of the effects individually. In IS literature, super-additive value synergies or benefits from synergy realization can often be seen in improved firm performance [26] or competitive advantage [22].

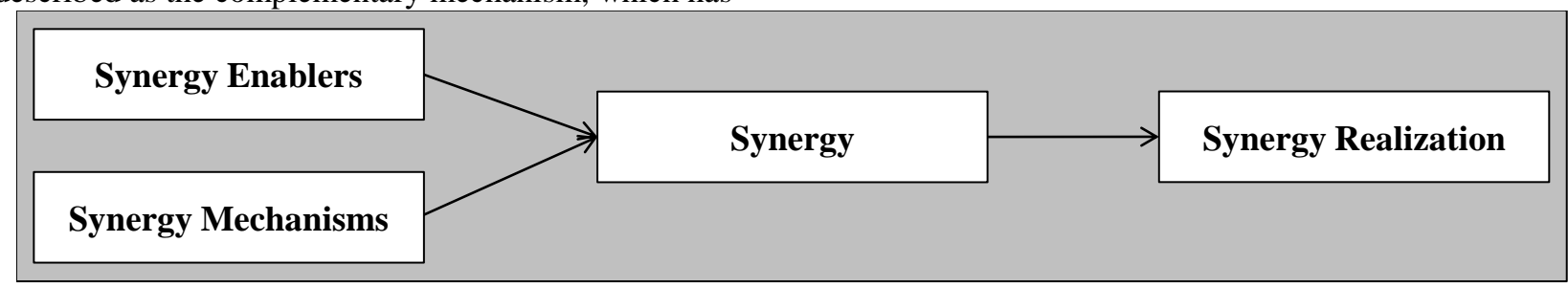

Figure 1. A holistic view of synergy in IS literature [6]

\section{Research Method}

As there are only a few previous findings that could be transferred to the context of synergistic interactions among data assets, I chose a two-step methodological approach. Step 1 is literature based and conceptual; step 2 follows an explorative, qualitative approach with semi-structured interviews and a thematic analysis.

In the first step, I proposed an initial conceptual framework for exploring synergistic relationships among data assets. I aimed to add clarity by proposing that data is a subcategory of IT resources and has unique characteristics that leverage synergistic effects more than other resources. I built the basis for my conceptual framework by means of a structured literature review in which I identified crucial characteristics of data and grouped them according to the general model of synergistic relationships used by Nevo and Wade [13] in an inductive way [30].

The resulting initial framework provides the input for the second step of my method in which I refine and contextualize the synergistic relationships among data assets. I do so by conducting semi-structured expert interviews with data experts to provide an empirical basis, because this kind of qualitative data gathering enables an in-depth understanding of research objects [31]. I based my interview evaluation and analysis on the well-established method of thematic analysis [32] that has also been applied in the data analytics context recently [33]. It enables the researcher to encode and analyze qualitative data by revealing and clustering themes, for example, patterns that can be further analyzed and mapped to gain an in-depth understanding of the research object. For this study, a thematic analysis is appropriate to identify, refine, and 
contextualize the enablers and mechanisms of synergy among data $[32,34]$.

I conducted 14 semi-structured interviews with experts in the field of data analytics that included data analysts, data scientists, and business intelligence experts. I selected data experts from various companies and varied the amount of experience they had working in managing information technologies (between 2 and 30 years) and their position in the company (from associate to director of analytics). This heterogeneous sample ensures a comprehensive portrait of data synergy. Following Schüritz et al. [35], I numbered the interview experts alphabetically in Greek letters from alpha (interview 1) to xi (interview 14). I used a semistructured interview guide, and the interviews lasted between 40 and 120 minutes. The interview guide was based on the synergy concept from literature. It included questions regarding enabling conditions and the requirements needed to combine and integrate data sources, illustrate the underlying mechanism to achieve value, and explain the associated additional business value derived from such data projects. The interviews were recorded and transcribed verbatim. For data triangulation, I collected secondary data (e.g., management reports, press releases, website information, etc.) about the benefits of big data analytics and coded the qualitative data with the software ATLAS.ti [34].

I used a theoretically based and rigorous iterative thematic analysis with latent theme development. I then coded the interview data with a specific research question in mind (theoretically based) with the goal of interpretively investigating the underlying idea of the interview data (latent themes) [32].

First, I familiarized myself with the interview data. Based on this, I generated initial codes by searching for recurring patterns in reference to my research question and initial synergy framework. Second, I grouped the initial codes into subthemes. Third, these subthemes were aggregated into main themes, which I revised and condensed repeatedly. The coding procedure was characterized by an ongoing review and analysis of relevant literature [32] and continuous iteration between the interview results and literature findings derived from my first methodological step. Regarding the synergy framework, the subthemes represented specific components of enablers and mechanisms, whereas the respective main themes represented the enabling conditions, synergistic mechanisms, and potential outcomes. Then these findings were mapped onto the initial synergy framework. Consequently, I was able to refine and contextualize the synergy factors among data assets.

\section{Towards a conceptual framework of synergy among data assets}

In a first step to address my research question, I synthesize relevant literature, deriving crucial synergy enablers, mechanisms and outcomes applied to the data context, to develop an initial conceptual model derived from literature.

In general, data assets comprise data and corresponding hardware (e.g. databases) as well as corresponding software [15]. Data assets can be understood as IT assets in companies with the ability to share or aggregate information [36]. Nevo and Wade [13] further define these data and IT assets as widely available, off-the-shelf, or commodity-like information technologies that are used to process, store, and disseminate information, which are often conceptualized as IT resources $[12,15]$. Therefore, I concluded that data and data assets are a subcategory of IT resources. Combining and synthesizing heterogeneous assets of data (e.g., various internal and external data sources, data from ERP and CRM systems, etc.) may lead to new insights and further value generation in organizations if their relationship is synergistic. Similar to the general concept of synergy, synergistic-related data assets refer to the additional return an organization can achieve from multiple data assets, which cannot be derived from isolated, standalone data sources [9].

I argue that data assets have unique characteristics with greater potential of leveraging synergy than other non-IT resources. I justify this argument by identifying and explaining crucial characteristics of data from various literature streams that facilitate the enabling conditions, mechanisms of data and their synergistic outcomes. I address five unique characteristics of data assets that are related to the potential of synergy enhancement. I argue that shareability, transportability, combinability, non-consumability, and versatility enable the mutual interaction between two or more data assets to a certain degree (see Table 2).

The shareability and almost instantaneous transportability of data simplifies the interaction of data from various information systems. Furthermore, their combinability means that heterogeneous data sources can easily be united into a single data asset whereby the original single data source becomes less important. In addition, data can be accessed and used simultaneously by many users without diminishing its information value for future usage (nonconsumability). 


\begin{tabular}{|l|l|l|}
\hline \multicolumn{3}{|c|}{ Table 2. Overview of typical characteristics of data assets } \\
\hline Characteristics & Explanation & References \\
\hline Shareability & $\begin{array}{l}\text { Data is sharable, not exchangeable; it can be given away and } \\
\text { retained at the same time. Several users can simultaneously use the } \\
\text { same unit of the resource. }\end{array}$ & {$[37]$} \\
\hline Transportability & Data is transportable virtually instantaneously over large distances. & {$[38,39]$} \\
\hline Combinability & $\begin{array}{l}\text { Data assets can be combined into a single asset and obscure } \\
\text { individual characteristics. }\end{array}$ & {$[38,40]$} \\
\hline $\begin{array}{l}\text { Non- } \\
\text { Consumability }\end{array}$ & $\begin{array}{l}\text { Usage or access does not diminish the value of the resource } \\
\text { available for future use. }\end{array}$ & {$[41]$} \\
\hline Versatility & $\begin{array}{l}\text { Data as a versatile resource can be used for a variety of purposes. It } \\
\text { is often versatile to an almost unlimited degree. }\end{array}$ & {$[42]$} \\
\hline
\end{tabular}

Depending on the specific topics and areas of application, data is a versatile resource and can be used for a variety of purposes [41]. These characteristics ensure that data assets "fit with each other [...] and are able to seamlessly work with each other" $[12$, p. 4$]$ to a certain extent and are therefore crucial subitems of the previous stated enabling condition, compatibility $[13,40,43]$.

Next to enabling conditions, certain synergistic mechanisms are important to leverage synergy effects in a data context. As stated previously, mechanisms are the activities that take place between resources (such as data assets) to realize their potential synergy $[12,13]$. Based on existing IS literature [14, 15], I recognize that the underlying synergistic mechanisms among data arise from differences among the data sources and the potential that these various sources can mutually support, reinforce, and enhance each other. This is often described as complementarity among resources [29].

Adapted from Wade and Hulland [44], mutual reinforcement means in this context that data assets consistently work with each other, support each other, and enhance each other's impact. The enabling condition, compatibility, and the complementary mechanism among heterogeneous data assets can lead to synergistic outcomes, which gives rise to the emergence of new value that cannot be reduced to individual assets [22]. As this new value of synergisticrelated data assets might be greater than the sum of their individual parts, their interaction can lead to super-additive value generation [14]. The data assets (a) and (b) enjoy super-additive value synergies if their joint value is larger than the sum of each data asset in isolation: Value (data asset $\mathrm{a}+$ data asset $\mathrm{b})>$ Value (data asset a) + Value (data asset b).

To summarize my findings, I am able to derive an initial conceptual framework of synergy among data assets from literature (Figure 3). Derived from various literature streams, my initial synergy framework consists of compatibility as a relevant enabler and complementarity as the synergy mechanism leading to super-additive value generation initiated through the interaction among various data assets.

\section{Synergy enabler}

Compatibility

- Shareability

- Transportability

- Combinability

- Non-Consumability

- Versatility

\begin{tabular}{l} 
Synergy mechanism \\
Complementarity \\
- Mutually reinforce and \\
enhance impact of each \\
other's data asset \\
\hline
\end{tabular}

Synergy among data

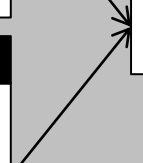

Synergy realization

Super-additive value

- Value of synergisticrelated data $>$ sum of individual parts

Figure 3. Initial conceptual model of synergistic relationships of data assets (derived from literature) 


\section{Empirical insights of synergy among data assets}

Based on the qualitative-empirical research approach that I conducted in the second step of the analysis and the continuous iteration between the interview results on the one hand, and findings from literature on the other hand, I revised my initial conceptual synergy framework from chapter 4. I identified and redefined several factors: synergy enablers, mechanisms, and synergy realization. I discuss the emergent results in turn.

\subsection{Synergy enablers}

The interview results reveal that there are two important enablers to consider in the context of data assets. First, as stated previously in step 1, data assets must consist of components that are compatible. Compatibility in a data context mainly refers to the condition that certain data properties (such as data format, common key, etc.) match and align. Second, to realize any kind of business value in an organization, the various data assets must include a contextual relation to each other. I derived these enabling conditions from the interviews (Table 3). (Note: many of the informants provided similar explanations; I include only a small selection for illustrative purposes.)

From the quotations it is clear that those circumstances, that support and enable the progress of synergistic interactions among data assets lead to two distinct but mutually supporting enablers.

Compatibility: In order to achieve the fit and alignment of heterogeneous data assets, they must have matching properties, for example, common data definitions, homogeneous data format for all assets, etc. This is often subsumed under data quality [e.g. 38]. It is just as important that the linked data assets are similar content-wise, in other words, a common key that allows connecting heterogeneous assets seamlessly is needed. This includes, according to one interviewee, "timestamps, product number, customer names etc." (Data Expert - Alpha). Furthermore, it became clear that the five specific characteristics of data assets (namely shareability, transportability, combinability, non-consumability, and versatility) support the compatibility among them: "Access and having a workable interface between two data systems is crucial in my opinion" (Data Expert - Delta). In other words, these properties allow data to be shared and transported between various data assets. Another informant stated that "[the] same data is used for various purposes in our company. [...] We can use it now and re-use it again in a couple of months" (Data Expert - Epsilon).
This statement supports the non-consumability of data and especially their versatility.

\begin{tabular}{|c|c|}
\hline \multicolumn{2}{|c|}{ Table 3. Synergy enablers among data assets } \\
\hline Compatibility & $\begin{array}{l}\text { "Every time data assets were not } \\
\text { compatible with each other, it was } \\
\text { mainly because of the different } \\
\text { format." (Data Expert - Epsilon) } \\
\text { "Any characteristics that show both } \\
\text { datasets identically, with which you } \\
\text { can compare something, for } \\
\text { example, temperature, product } \\
\text { number, or customer number, etc." } \\
\text { (Data Expert - Alpha) }\end{array}$ \\
\hline $\begin{array}{l}\text { Contextual } \\
\text { Relatedness }\end{array}$ & $\begin{array}{l}\text { "Depending on the context, there is } \\
\text { a greater or smaller added value. An } \\
\text { advantage can arise if you combine } \\
\text { production data from a machine hall } \\
\text { with weather information. This will } \\
\text { not be the case if you add completely } \\
\text { different data (such as survey } \\
\text { data)." (Data Expert - Alpha) } \\
\text { "They must have something in } \\
\text { common-content-wise and } \\
\text { contextual." (Data Expert - Alpha) }\end{array}$ \\
\hline
\end{tabular}

Contextual Relatedness: Upon reflection, the analysis of our interviews makes clear that the compatibility of data assets alone does not describe entirely the enabling conditions needed to derive synergistic value from two or more interacting data sets. Having a contextual relatedness among the data assets is at least as important. "Just linking various data sets, such as football results, at a certain date with sensory data from our manufacturing hall will be less effective, the various data sets should be in the same context" (Data Expert - Lambda). These quotations, along with other interview results, make it clear that the interaction of various data assets leads to additional value only when the unifying partial information can be subsumed in a specific context, or as one interviewee stated: "the linkage needs to makes sense logically to lead to additional value, for example, support a use case or the tasks and functions of a division" (Data Expert - Alpha).

\subsection{Synergy mechanism}

To better understand the underlying mechanisms that take place among data assets and how they lead to additional value, I turn to the interviews. It became clear from my interviews that the activities that take place among data assets to realize their potential synergy effect are on an informational level. While 
data assets interact with each other, they give away and retain information at the same time (exchange) and thus can enrich their information content, which I summarize as informational complementarity. Before describing this mechanism in more detail, I provide examples of how informants explained the potential complimentary mechanism of combining data assets:

"The more information I have, the better I can classify and create the offer to serve the customer in a personalized and individualized way. So that he meets his needs through us exactly at that point in time and in that moment. [...] One can form the context." (Data Expert - Iota)

"It gets clear with the context of data sources. [...] Data contain information, but no data set has the information entirety. The interaction of more data increases the information entirety and [...] thereby generates an added value." (Data Expert - Theta).

These quotations indicate that the reinforcing mechanism is present on an informational level. When data assets are combined, these assets enlarge and complement the impact of the other data assets. One manager emphasized this by stating: "The interaction often reveals mutual thematic dependencies between variables and sets of information" (Data Expert Gamma).

Furthermore, the results show that combining and synthesizing various data assets typically enriches the informational content and leverages the explanatory power of a specific subject. The basic assumption is that in most cases a data source contains partial information on a specific subject or topic, for example, "sales history for each customer" (Data Expert - Mu). Here the specific subject is the customer and the partial information of sales history. Combining this partial information with further data sources, which are also partial, for example, "historical weather information at the residence of the customer" (Data Expert - Mu), enlarges the overall informativeness of a specific subject, for example, purchasing behavior of a customer and its dependency on external factors.

\subsection{Synergy realization}

The revised enabling conditions compatibility and contextual relatedness, as well as the informational complementary mechanism, can lead to synergistic outcomes among data assets. The interviews, in addition, revealed that synergistic-related data assets enhance the information value of a specific domain and realize synergy effects by leading to a more precise and "accurate picture of the reality" (Data Expert - Zeta). Due to this fact, and referencing Tanriverdi [14], I subsume the synergy realization among data assets as super-additive information value generation. The experts described the synergy realization in the following manner:

"Data are basically a depiction of the reality. Through its interaction we can portray reality much better." (Data Expert - Alpha)

"By combining data, one normally gets from a onedimensional to a multidimensional view" (Data Expert - Delta)

The synergistic interaction of two or more heterogeneous data assets enhances the overall informativeness and complements the depiction of a certain work domain. A specific subject is not only observed from one dimension, rather, the data's information is multi-dimensional. The increased informativeness allows, as one interviewee said, a "more realistic projection of the topic or thing I want to analyze in my organization" (Data Expert - Kappa). Another interviewee gave an example of this: " $B y$ combining data from our CRM system and online behavioral data from our e-commerce shop we are able to observe the purchasing behavior of our customers from various perspectives [...] such as the places of residence, weekdays, personal interests etc. [...] to recommend [to] him or her products that the user is more willing to buy" (Data Expert - Iota). Thus, the synergistic interaction of two data assets (in this case, a CRM system and an e-commerce system) substantially enhances the information value of a specific domain (customer behavior data) and makes possible an examination of a certain subject (such as the customer of an organization) from various perspectives.

\section{Discussion}

With advances in IT technologies, the volume and variety of data being captured and stored has increased extensively. Combining data from various sources can make it more feasible for organizations to make datadriven decisions, to personalize products, or to sell data.

The goal of this study was to examine the specific context of synergistic interactions between heterogeneous data assets and to explain how combined data sources generate additional value.

I propose a generic conceptual framework of enabling conditions, mechanisms, and realization to better understand the synergistic interaction among data assets. Anchored in systems theory [13], synergy between various data assets is realized using specific enablers and mechanisms. The results, based on literature and 14 expert interviews, show that compatibility and contextual relatedness are the two crucial enabling conditions that facilitate the 
interaction among data assets. Five unique characteristics of data (shareability, transportability, combinability, non-consumability, and versatility) enable to a substantial degree the mutual interaction of data assets and can be understand as important subitems of compatibility. Grounded in the economic theory of complementarities [29] and further detailed in the interview process, I identified the mechanism that takes place among data assets to realize their potential synergy effect as the informational complementary mechanism.

The enabling conditions and informational complementarity together drive the synergistic interaction among data assets, leading to super-additive information values in organizations. This means that the emergent informativeness is greater than the sum of the value of each data asset in isolation. Figure 4 is an illustration of the revised conceptual model of synergistic relationships between data assets and answers my research question of how the synergistic interaction among data assets can be conceptualized.

In the exploration of the synergistic relationships among data assets, it became clear that all my interview partners stressed the importance of the contextual relatedness of potential linked data assets to achieve additional value. Only if both enabling conditions, namely compatibility and contextual relation, are met, will the interaction among various data assets result in outcomes that are greater than the sum of the value of their individual data parts (superadditive information value). Conversely, incompatible data assets do not support synergistic interactions. Rather, they must be modified substantially before complementary mechanisms can be observed.

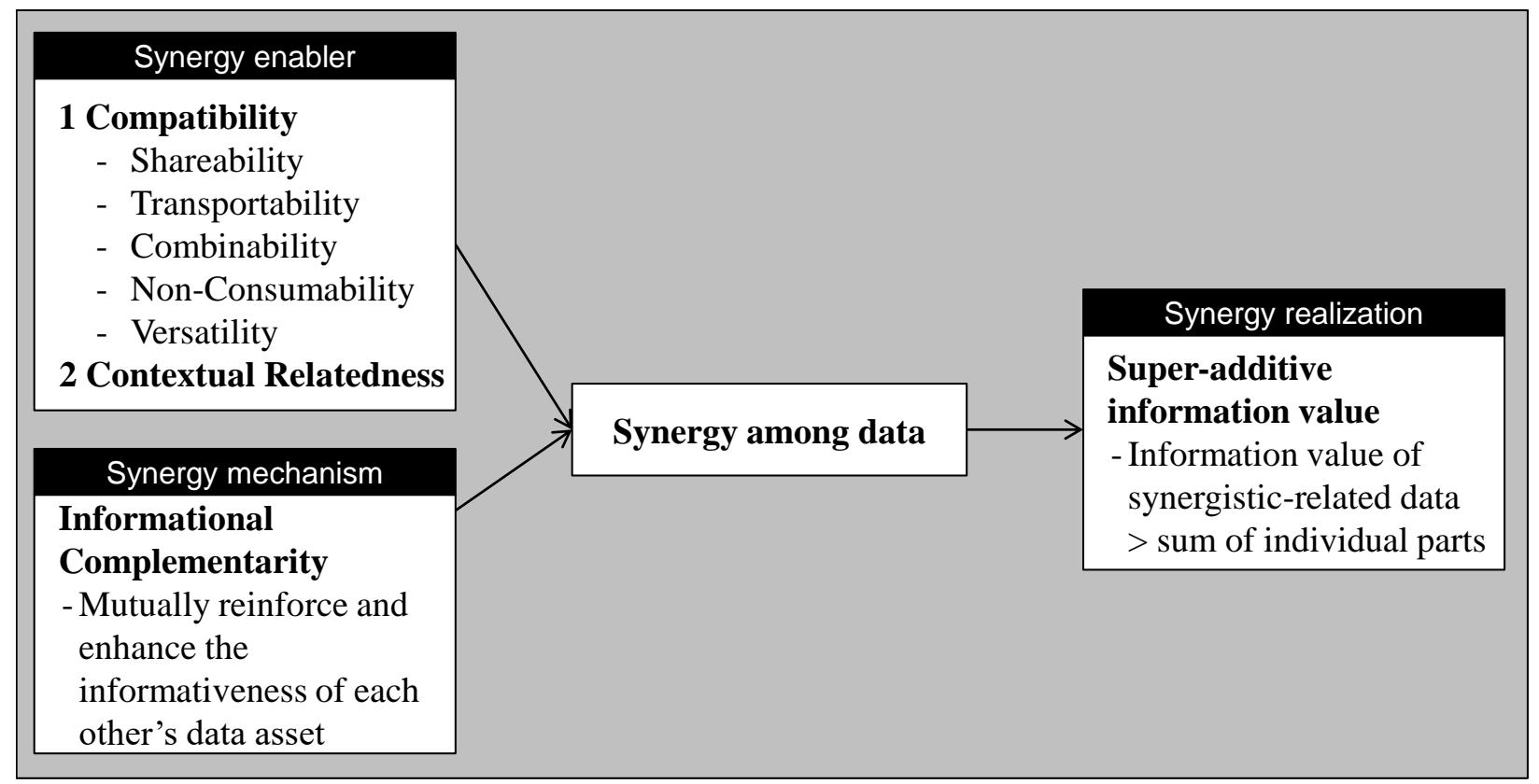

Figure 4. Revised conceptual model of synergistic relationships of data assets

7. Contribution, limitations, and future research

\subsection{Contribution}

For IS researchers, this study has a number of implications. First, I have extended the big data value literature by adapting the important but rarely applied concept of synergy to the data context. Existing studies have mainly focused on synergy effects at an interorganizational level [26], between organizational resources [14], or between technologies and individuals [13], whereas I further contributed to the synergy literature by shedding light on synergistic interactions on a data and informational level of analysis. The study sheds light on the theorization of business value from data variety. The synergistic interaction among data helps to understand how the synthesis of various data assets leads to business value in firms.

Second, the study extends the body of knowledge of synergistic enablers. To the best of my knowledge, the necessary contextual relatedness of resources as an enabling condition has not received appropriate attention in the current synergy literature. Furthermore, 
my paper shows that data are a major source of synergies due to their unique characteristics (shareability, transportability, combinability, nonconsumability, and versatility), enabling mutual interaction to a remarkable degree.

Third, this study sheds new light on the complementarity theory. Studies exploring this perspective often focus on functional complementarities between IT resources and business resources. They argue that additional value is leveraged by enlarging and complementing the efficiency and functionality between IT and business resources [14, 45]. This study reveals that complementarities among data assets can be seen as an informational complimentary effect, by enlarging the informativeness and explanatory power of data.

For IS practitioners, the study provides a systematic means of understanding the importance of synergistic interaction of various data sources. My synergy framework, including enablers, mechanisms, and synergy realization, can help practitioners in creating synergy among various data sources and data sets and increase their informativeness, and as a result, organizational effectiveness and efficiency. Considering these factors, I paid attention to the potential for developing a synergistic relationship among heterogeneous data sources; by leveraging it, organizations are able to continuously transform themselves into data-driven companies. My synergy framework can also be used to shed light on decisions about which new data sources should be interconnected with existing information systems in organizations. Referring to the synergy enablers, new data sources have to be compatible with existing systems and their partial information has to have a contextual relationship with the stored information of the existing system.

\subsection{Limitations and future research}

There are two limitations of my study that could stimulate further research. First, although I considered extant literature as well as empirical insights, I cannot guarantee to have covered all possible synergy factors and characteristics. Future research would involve more in-depth empirical approaches, such as case studies and other research methods, to understand the synergistic relationships among data assets in detail, as well as increasing the initial sample size of 14 expert interviews.

Second, this study focused specifically on synergy at a data and informational level of analysis, and further research is required to understand how and why these synergistic interactions among data assets lead to emergent business value in various domains (e.g., procurement, sales, etc.) and industries (e.g., retail, automotive, energy sector, etc.). Future empirical studies may also, in their analysis, shed light on potential negative synergy effects [17] regarding interacting data assets.

All these results planned for future research would add to knowledge on the business value of big data for both practitioners and researchers. Furthermore, it would deepen the understanding of the synergy concept in IS research.

\section{References}

[1] Chen, H., Chiang, R.H., and Storey, V.C., "Business Intelligence and Analytics: From Big Data to Big Impact", MIS Quarterly, 36(4), 2012, pp. 1165-1188.

[2] Weibl, J., and Hess, T., "Success or Failure of Big Data: Insights of Managerial Challenges from a Technology Assimilation Perspective", Proceedings of the Multikonferenz Wirtschaftsinformatik (MKWI), 2018, pp. 12-59.

[3] Mcafee, A., Brynjolfsson, E., and Davenport, T.H., "Big data: the management revolution", Harvard Business Review, 90(10), 2012, pp. 60-68.

[4] Goes, P.B., "Editor's comments: big data and IS research", MIS Quarterly, 38(3), 2014, pp. iii-viii.

[5] Henke, N., Bughin, J., Chui, M., Manyika, J., Saleh, T., Wiseman, B., and Sethupathy, G., "The Age of Analytics: Competing in a Data-Driven World", in: McKinsey Global Institute, London, 2016, pp. 1-125.

[6] Lycett, M., "'Datafication': making sense of (big) data in a complex world", European Journal of Information Systems, 22(4), 2013, pp. 381-386.

[7] Seddon, P.B., Constantinidis, D., and Dod, H., "How does business analytics contribute to business value?", Proceedings of the 33rd International Conference on Information Systems, 2012

[8] Wixom, B.H., and Watson, H.J., "An empirical investigation of the factors affecting data warehousing success", MIS Quarterly, 25(1), 2001, pp. 17-41.

[9] Günther, W.A., Mehrizi, M.H.R., Huysman, M., and Feldberg, F., "Debating Big Data: A Literature Review on Realizing Value from Big Data", The Journal of Strategic Information Systems, 26(3), 2017, pp. 191-209.

[10] Malgonde, O., and Bhattacherjee, A., "Innovating using big data: A social capital perspective", Proceedings of the 20th Americas Conference on Information Systems, 2014, pp. 1-9.

[11] Arisa, S., and D., G.R., "Towards an understanding of the role of business intelligence systems in organisational knowing", Information Systems Journal, 26(4), 2016, pp. 339-367.

[12] Someh, I.A., and Shanks, G., "The role of synergy in achieving value from business analytics systems", Proceedings of the 34th International Conference on Information Systems, 2013, pp. 1-16.

[13] Nevo, S., and Wade, M., "The formation and value of IT-enabled resources: antecedents and consequences of 
synergistic relationships", MIS Quarterly, 34(1), 2010, pp. 163-183.

[14] Tanriverdi, H., "Performance effects of information technology synergies in multibusiness firms", MIS Quarterly, 30(1), 2006, pp. 57-77.

[15] Aral, S., and Weill, P., "IT assets, organizational capabilities, and firm performance: How resource allocations and organizational differences explain performance variation", Organization Science, 18(5), 2007, pp. 763-780.

[16] Griffith, T.L., Sawyer, J.E., and Neale, M.A., "Virtualness and knowledge in teams: Managing the love triangle of organizations, individuals, and information technology", MIS Quarterly, 27(2), 2003, pp. 265-287.

[17] Titah, R., and Barki, H., "Nonlinearities between attitude and subjective norms in information technology acceptance: a negative synergy?", MIS Quarterly, 33(4), 2009, pp. 827-844.

[18] Barney, J., "Firm resources and sustained competitive advantage", Journal of management, 17(1), 1991, pp. 99-120.

[19] Ackoff, R.L., "Towards a system of systems concepts", Management Science, 17(11), 1971, pp. 661-671.

[20] Churchman, C.W., The Design of Inquiring Systems: Basic Concepts of Systems and Organization, Basic Books, Inc, New York, 1971.

[21] Kast, F.E., and Rosenzweig, J.E., "General systems theory: Applications for organization and management", Academy of Management Journal, 15(4), 1972, pp. 447-465.

[22] Nevo, S., and Wade, M., "Firm-level benefits of ITenabled resources: A conceptual extension and an empirical assessment", The Journal of Strategic Information Systems, 20(4), 2011, pp. 403-418.

[23] Grover, V., and Kohli, R., "Cocreating IT value: New capabilities and Metrics for Multifirm Environments", MIS Quarterly, 36(1), 2012, pp. 225-232.

[24] Tanriverdi, H., "Information technology relatedness, knowledge management capability, and performance of multibusiness firms", MIS Quarterly, 29(2), 2005, pp. 311334.

[25] Ferratt, T.W., Prasad, J., and Enns, H.G., "Synergy and its limits in managing information technology professionals", Information Systems Research, 23(4), 2012, pp. 1175-1194.

[26] Venkatesh, V., and Bala, H., "Adoption and impacts of interorganizational business process standards: Role of partnering synergy", Information Systems Research, 23(4), 2012, pp. 1131-1157.

[27] Sarkar, M.B., Echambadi, R., Cavusgil, S.T., and Aulakh, P.S., "The influence of complementarity, compatibility, and relationship capital on alliance performance", Journal of the Academy of Marketing Science, 29(4), 2001, pp. 358-373.

[28] Dyer, J.H., and Singh, H., "The relational view: Cooperative strategy and sources of interorganizational competitive advantage", Academy of Management Review, 23(4), 1998, pp. 660-679.

[29] Milgrom, P., and Roberts, J., "Complementarities and fit strategy, structure, and organizational change in manufacturing", Journal of Accounting and Economics, 19(2), 1995, pp. 179-208.
[30] Tilly, R., Posegga, O., Fischbach, K., and Schoder, D., "Towards a Conceptualization of Data and Information Quality in Social Information Systems", Business \& Information Systems Engineering, 59(1), 2017, pp. 3-21.

[31] Myers, M.D., and Newman, M., "The Qualitative Interview in IS Research: Examining the Craft", Information and Organization, 17(1), 2007, pp. 2-26.

[32] Braun, V., and Clarke, V., "Using thematic analysis in psychology", Qualitative Research in Psychology, 3(2), 2006, pp. 77-101.

[33] Shanks, G.G., Bekmamedova, N., and Willcocks, L.P., "Business Analytics: Enabling Strategic Alignment and organisational Transformation", Proceedings of the 20th European Conference on Information Systems, 2012

[34] Miles, M.B., Huberman, A.M., and Saldana, J., Qualitative Data Analysis - A Methods Sourcebook, Sage Publications, 3rd, Thousand Oaks, 2013.

[35] Schüritz, R.M., Seebacher, S., Satzger, G., and Schwarz, L., "Datatization as the Next Frontier of Servitization Understanding the Challenges for Transforming Organizations", Proceedings of the 38th International Conference on Information Systems, 2017

[36] Goodhue, D.L., Wybo, M.D., and Kirsch, L.J., "The Impact of Data Integration on the Costs and Benefits of Information Systems", MIS Quarterly, 16(3), 1992, pp. 293311.

[37] Pipino, L.L., Lee, Y.W., and Wang, R.Y., "Data quality assessment", Communications of the ACM, 45(4), 2002, pp. 211-218.

[38] Nelson, R.R., Todd, P.A., and Wixom, B.H., "Antecedents of information and system quality: an empirical examination within the context of data warehousing", Journal of Management Information Systems, 21(4), 2005, pp. 199235.

[39] Eaton, J.J., and Bawden, D., "What kind of resource is information?", International Journal of Information Management, 11(2), 1991, pp. 156-165.

[40] Wang, R.Y., and Strong, D.M., "Beyond accuracy: What data quality means to data consumers", Journal of Management Information Systems, 12(4), 1996, pp. 5-33.

[41] Levitin, A.V., and Redman, T.C., "Data as a resource: properties, implications, and prescriptions", MIT Sloan Management Review, 40(1), 1998, pp. 89.

[42] Gorla, N., Somers, T.M., and Wong, B., "Organizational impact of system quality, information quality, and service quality", The Journal of Strategic Information Systems, 19(3), 2010, pp. 207-228.

[43] Kappelman, L., Mclean, E., Johnson, V., and Gerhart, N., "The 2014 SIM IT key issues and trends study", MIS Quarterly Executive, 13(4), 2014, pp. 237-263.

[44] Wade, M., and Hulland, J., "The resource-based view and information systems research: Review, extension, and suggestions for future research", MIS Quarterly, 28(1), 2004, pp. 107-142.

[45] Brynjolfsson, E., and Hitt, L.M., "Beyond computation: Information technology, organizational transformation and business performance", The Journal of Economic Perspectives, 14(4), 2000, pp. 23-48. 